\title{
Fragility of the A-type AF and CE Phases of Manganites: An Exotic Insulator-to-Metal Transition Induced by Quenched Disorder
}

\author{
G. Alvarez, ${ }^{1}$ H. Aliaga, ${ }^{2,3}$ C. Şen, ${ }^{4}$ and E. Dagotto ${ }^{2,3}$ \\ ${ }^{1}$ Computer Science 83 Mathematics Division, Oak Ridge National Laboratory, Oak Ridge, TN 37831 \\ ${ }^{2}$ Condensed Matter Sciences Division, Oak Ridge National Laboratory, Oak Ridge, TN 32831 \\ ${ }^{3}$ Department of Physics and Astronomy, The University of Tennessee, Knoxville, TN 37996 \\ ${ }^{4}$ National High Magnetic Field Laboratory and Department of Physics, Florida State University, Tallahassee, FL 32310
}

\begin{abstract}
Using Monte Carlo simulations and the two $e_{\mathrm{g}}$-orbital model for manganites, the stability of the $\mathrm{CE}$ and A-type antiferromagnetic insulating states is analyzed when quenched disorder in the superexchange $J_{\mathrm{AF}}$ between the $t_{2 \mathrm{~g}}$ localized spins and in the on-site energies is introduced. At vanishing or small values of the electron-(Jahn-Teller)phonon coupling, the previously hinted "fragility" of these insulating states is studied in detail, focusing on their charge transport properties. This fragility is here found to induce a rapid transition from the insulator to a (poor) metallic state upon the introduction of disorder. A possible qualitative explanation is presented based on the close proximity in energy of ferromagnetic metallic phases, and also on percolative ideas valid at large disorder strength. The scenario is compared with previously discussed insulator-to-metal transitions in other contexts. It is argued that the effect unveiled here has unique properties that may define a new class of giant effects in complex oxides. This particularly severe effect of disorder must be present in other materials as well, in cases involving phases that arise as a compromise between very different tendencies, as it occurs with striped states in the cuprates.
\end{abstract}

PACS numbers: 75.30.Mb, 75.10.Lp, 75.47.-m

\section{INTRODUCTION}

The understanding of transition metal oxides is one of the most important open challenges in Condensed Matter Physics. Exotic and complex phenomena emerge from the correlated nature of carriers in these materials, such as high critical temperature superconductivity and colossal magnetoresistance (CMR) $1,2,3,4,5,6,7,8,9,10,11,12,13,14,15,16,17$ In recent years, much progress has been made in this context after the realization that several competing states, with quite different properties, are very close in energy in these compounds. As a consequence, small variations in carrier density, temperature, pressure, magnetic fields, and other variables often lead to giant responses and an intrinsic electronic softness $\stackrel{2}{2}$ Moreover, the effect of quenched disorder appears to be important to understand the CMR effect, according to recent theoretical and experimental investigations briefly reviewed below. This combination of strong correlation and disorder effects, and the simultaneous relevance of several degrees of freedom such as spin, charge, orbital, and lattice, lead to a plethora of unusual properties, with tremendous potential for functionalities.

In this paper, using theoretical models and Monte Carlo simulations, the unexpected giant responses of some insulating states to the introduction of quenched disorder are discussed. Recent investigations by Aliaga et $a l$ 18 already briefly described a curious "fragility" of the insulating charge-ordered and orbital-ordered CE state of half-doped manganites. In those previous investigations the term "CE glass" was coined, and it was observed that the spin correlations at large distances characteristic of the $\mathrm{CE}$ state rapidly decrease in mag- nitude by increasing the strength of the quenched disorder in the links, namely by adding a random component to the superexchange $J_{\mathrm{AF}}$ between the $t_{2 \mathrm{~g}}$ spins degrees of freedom. In independent studies, Motome et $a l \stackrel{19}{19}$ also observed that disorder suppresses charge order more strongly than ferromagnetic order using a one-band model for Mn oxides (see also Sen et $a l^{20}$ ). These conclusions appear to be potentially important since experimental investigations by Akahoshi et al ${ }^{21}$ and Nakajima et $a l^{22}$ have observed that for a manganite material in the clean limit (namely, with minimal quenched disorder) magnetoresistance effects are absent. However, by introducing quenched disorder (via a random distribution of trivalent ions in the crystal) then a clear CMR effect is observed. Thus, experimental evidence suggests that quenched disorder is vital to understand the manganites. This is in agreement with the early theoretical investigations of Burgy et al. using simple "toy" models with two states in competition, supplemented by random resistor

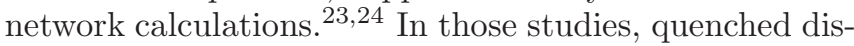
order was needed to obtain a CMR effect at low fields.

In the investigations presented in this manuscript, we have studied in considerable detail and using larger lattices the fragility effect first noticed in Refs. 18 19.20. The focus of the investigations is on the half-doped regime of manganites, where several previous efforts have established the phase diagrams with good accuracy. In particular, it is known that a $\mathrm{CE}$ phase is clearly stabilized at intermediates values of $J_{\mathrm{AF}}$. Of much importance for the discussion below is that this phase exists independently of the value of the electron-(JahnTeller)phonon coupling $\lambda$. Such a curious observation was understood independently by at least two groups ( $e$.

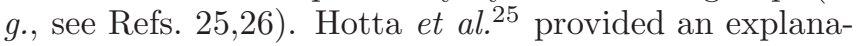


tion where the well-known zigzag chains of the CE arrangement arise from an optimization process between two competing tendencies: the double exchange mechanism that favors ferromagnetism and the coupling between the localized spins $J_{\mathrm{AF}}$ that favors antiferromagnetism. This competition leads to an exotic insulator, the $\mathrm{CE}$ state, in a relatively narrow region of parameter space, interpolating between the more conventional ferromagnetic (FM) and antiferromagnetic (AF) regimes. Ferromagnetic one-dimensional zigzag chains spontaneously emerge, with antiferromagnetic coupling between these chains. Moreover, the insulating nature of this state arises from the unexpected band insulator properties of the zigzag chains at $n=0.5$. This combination of frustrational and geometrical factors leads to a stable insulating $\mathrm{CE}$ state. However, with hindsight clearly its nature can be anticipated to be quite fragile since its stability is induced by competition of very different tendencies.

In our investigations, we have studied the CE state in two (2D) and three (3D) dimensions, as well as another insulating state, the A-type AF state that is stable only in 3D. Small amounts of quenched disorder were introduced and we have analyzed in detail the influence of this disorder over the transport properties of the resulting states, using canonical techniques $\stackrel{27}{\stackrel{27}{2}}$ To our surprise, a rapid transition from an insulator to a poor metal was observed. This effect appears robust: it is similar in both $2 \mathrm{D}$ and $3 \mathrm{D}$, and both for the $\mathrm{CE}$ and $\mathrm{A}$ type insulators, to the extent that the electron-phonon coupling is small. In other words, it is the "topological" CE insulator unveiled in previous investigations ${ }^{25}$ that shows the strongest fragility toward quenched disorder. This CE state is stabilized neither by a very strong nearest-neighbors Coulomb repulsion nor by a very strong electron-phonon coupling, but by more subtle effects related to frustration and geometry, thus causing its fragility. Also, these states appear to be highly susceptible to the introduction of magnetic fields, as also discussed in the text.

How exotic is the insulator-metal transition found here when analyzed in a more general framework? Anderson localization transitions can be induced via the introduction of disorder in a metal. In this case, quenched disorder transforms the metal into an insulator, which is the opposite of the transition we aim to discuss in this paper. Recent investigations on the effects of correlated disorder have shown that the metallic state can survive even in one dimensional systems ${ }^{28}$ but still the quenched disorder always favors the insulating tendencies, which does not happen in our case. In general, the appearance of a metal upon introducing disorder in an insulator is not a common phenomenon if carried out at constant electronic density. For instance, it is known that insulator to metal transitions appear in conventional semiconductors. But they occur via doping and the concomitant creation of an impurity band. The transmission of charge in disordered materials sometimes occurs via tunneling between large conducting regions $\stackrel{29}{\underline{2}}$ Doped polyacetylene has a similar behavior, although the metallic phase is generated via a qualitatively different process ${ }^{30} \mathrm{~A}$ similar phenomenon occurs in other transition metal oxides, such as the high temperature superconductors, where chemical doping introduces mobile carriers in the system, rendering the compound metallic or even superconducting ${ }^{2}$ In all these materials, carrier doping away from the electronic density corresponding to the insulator is needed to achieve the metallic state. This is not the case of the study reported in this paper that is carried out at a constant electronic density $n$.

Working at fixed $n$, an insulator to metal transition can occur also if uniaxial stress increases the overlaps between otherwise localized electronic states. Our analysis is not of this variety, since the hopping amplitudes (the model parameters which are the most susceptible to pressure) are left untouched in our investigations.

However, there are simple ways in which a disorderinduced insulator to metal transition can be envisioned and it can occur by the mere broadening of states by disorder. As the disorder strength increases, the gap will tend to close. Examples of this behavior were discussed by Dobrosavljević and collaborators $\stackrel{31.32}{\longleftarrow}$ where dynamical mean-field theory calculations unveiled a metallic regime in between Mott insulator and Anderson localized regions, at values of the disorder strength comparable to the Hubbard repulsion. However, we believe that this simple picture cannot be the full explanation of the numerical results reported here since the metallic tendencies appear even for small values of the disorder strength. In the study of Ref. 31 small amounts of disorder do not alter substantially the properties of the Mott insulator, but the reciprocal occurs: small values of the Hubbard $\mathrm{U}$ can rapidly transform an insulator to a metal at intermediate values of the disorder strength. Moreover, the subtle physics of zigzag chains is difficult to capture with mean-field approximations. An explanation of the CE fragility as given in Ref. 20 for the one-orbital model results of Ref. 19 is also based on the simple broadening of peaks idea. However, note that the $\mathrm{CE}$ state of halfdoped manganites at vanishing electron-phonon coupling seems more fragile than other insulators, and we believe that a more sophisticated explanation of its insulator to metal transition will be needed.

Finally, to further motivate these investigations it is important to remark that there is evidence suggesting that the CE state of the manganites may actually be of the exotic "topological" variety discovered by Hotta et $a l .25$ In fact, recent experimental efforts ${ }^{33.34}$ have challenged the common folklore that the $\mathrm{CE}$ state contains ions with sharply-defined charge $3+$ and $4+$, since no evidence of strong charge order was observed. A possible explanation of this puzzle is provided in Fig. 10 of Ref. 18 where the charge disproportionation between the even and odd sites of the CE state is shown as a function of $\lambda$. It was there observed that the regime of small $\lambda$ is the only one compatible with the new experiments. This is also in agreement with the well known fact that a fer- 
romagnetic metallic state must be very close in energy to the CE insulating states, since experimentally the latter is easily destabilized by a magnetic field into the former. At large $\lambda$, the studies in Ref. 18 showed that all states (FM, CE, and AF) are charge ordered, in contradiction with experiments. Thus, it is conjectured here that the fragility of the CE state may be of key relevance to understanding the manganites. Recent experimental results are compatible with this conclusion, namely quenched disorder affects the $\mathrm{CE}$ phase more aggressively than the FM state $\underline{35}$

If we accept that the close proximity of states with different properties (such as a metal or superconductor and an insulator) is the basic cause of the effect, then it follows that the giant effect of quenched disorder should be present in many other systems as well. For instance, the famous stripes ${ }^{36.37}$ in cuprates have recently been found to appear at intermediate narrow coupling regimes of phenomenological models for the superconductorantiferromagnetism competition 38.39 namely they are also a compromise between very different states. As a consequence, we here conjecture that $\mathrm{Cu}$-oxide stripes should also be highly susceptible to the explicit introduction of disorder. This conclusion can be extended to any intermediate narrow phase of transition metal oxides that originates from competing tendencies.

The organization of this manuscript is the following. In Section II, the model is described. Since this model has been extensively used in previous investigations, the discussion is brief. Section III contains the main results, namely the influence of link disorder on the transport properties of the system. The analysis is carried out for two insulating states (the $\mathrm{CE}$ and A-type $\mathrm{AF}$ phases) and in both two and three dimensions. Evidence for the metalization of the system upon the introduction of link quenched disorder is presented. Section IV describes the results for transport now including magnetic fields. Section V deals with on-site disorder, Section VI provides evidence that our results are not dramatically altered by increasing lattice sizes, and finally conclusions are provided in Section VII.

\section{MODEL AND DETAILS OF THE SIMULATION}

The standard double-exchange two-orbital model for manganites was used in this study. Since the model has been widely described in previous investigations, $\frac{\sqrt{\underline{\underline{n}}}}{\text { this }}$ section is brief. The two orbitals arise from the $e_{\mathrm{g}}$ states that are active at the Mn ions in Mn-oxides, as extensively discussed before 5.13 .14 The electronic density will be $n=0.5$ in all the results reported here. This corresponds to one electron per site of a square or cubic lattice, and it is the density where the CE state of manganites is experimentally known to be stable.

In more detail, the Hamiltonian for the two-orbital model is

$$
\begin{aligned}
H & =\sum_{\gamma, \gamma^{\prime}, i, \alpha} t_{\gamma \gamma^{\prime}}^{\alpha} \mathcal{S}\left(\theta_{i}, \phi_{i}, \theta_{i+\alpha}, \phi_{i+\alpha}\right) c_{i, \gamma}^{\dagger} c_{i+\alpha, \gamma^{\prime}} \\
& +\lambda \sum_{i}\left(Q_{1 i} \rho_{i}+Q_{2 i} \tau_{x i}+Q_{3 i} \tau_{z i}\right)+\sum_{i} \sum_{\alpha=1}^{\alpha=3} D_{\alpha} Q_{\alpha i}^{2} \\
& +\sum_{i, \alpha} J_{\mathrm{AF}}(i, \alpha) \vec{S}_{i} \cdot \vec{S}_{i+\alpha}+\vec{B} \cdot \sum_{i} \vec{S}_{i}
\end{aligned}
$$

where the factor that renormalizes the hopping in the $J_{\mathrm{H}} \rightarrow \infty$ limit is

$$
\begin{aligned}
\mathcal{S}\left(\theta_{i}, \phi_{i}, \theta_{j}, \phi_{j}\right) & =\cos \left(\frac{\theta_{i}}{2}\right) \cos \left(\frac{\theta_{j}}{2}\right) \\
& +\sin \left(\frac{\theta_{i}}{2}\right) \sin \left(\frac{\theta_{j}}{2}\right) e^{-i\left(\phi_{i}-\phi_{j}\right)}
\end{aligned}
$$

and the angles are used to parametrize the $t_{2 \mathrm{~g}}$ classical spins at every site. The parameters $t_{\gamma \gamma^{\prime}}^{\alpha}$ are the hopping amplitudes between the orbitals $\gamma$ and $\gamma^{\prime}$ in the direction $\alpha$. In two dimensions, these are $t_{a a}^{x}=-\sqrt{3} t_{a b}^{x}=$ $-\sqrt{3} t_{b a}^{x}=3 t_{b b}^{x}=1$, and $t_{a a}^{y}=\sqrt{3} t_{a b}^{y}=\sqrt{3} t_{b a}^{y}=3 t_{b b}^{y}=1$ and in three dimensions one needs also consider $t_{a a}^{z}=$ $t_{a b}^{z}=t_{b a}^{z}=0$ and $t_{b b}^{z}=4 / 3$.

Although the phononic coupling is barely used in the effort reported here, for completeness a description if provided. $Q_{1 i}, Q_{2 i}$ and $Q_{3 i}$ are normal modes of vibration that can be expressed in terms of the oxygen coordinate $u_{i, \alpha}$ as:

$$
\begin{aligned}
Q_{1 i} & =\frac{1}{\sqrt{3}}\left[\left(u_{i, z}-u_{i-z, z}\right)+\left(u_{i, x}-u_{i-x, x}\right)\right. \\
& \left.+\left(u_{i, y}-u_{i-y, y}\right)\right], \\
Q_{2 i} & =\frac{1}{\sqrt{2}}\left(u_{i, x}-u_{i-x, x}\right), \\
Q_{3 i} & =\frac{2}{\sqrt{6}}\left(u_{i, z}-u_{i-z, z}\right)-\frac{1}{\sqrt{6}}\left(u_{i, x}-u_{i-x, x}\right) \\
& -\frac{1}{\sqrt{6}}\left(u_{i, y}-u_{i-y, y}\right) .
\end{aligned}
$$

Also, $\quad \tau_{x i}=c_{i a}^{\dagger} c_{i b}+c_{i b}^{\dagger} c_{i a}, \quad \tau_{z i}=c_{i a}^{\dagger} c_{i a}-c_{i b}^{\dagger} c_{i b}, \quad$ and $\rho_{i}=c_{i a}^{\dagger} c_{i a}+c_{i b}^{\dagger} c_{i b}$. The constant $\lambda$ is the electronphonon coupling related to the Jahn-Teller distortion of the $\mathrm{MnO}_{6}$ octahedron $\stackrel{\underline{5}}{\underline{\underline{n}}}$ Regarding the phononic stiffness, and in units of $t_{a a}^{x}=1$, the $D_{\alpha}$ parameters are $D_{1}=1$ and $D_{2}=D_{3}=0.5$, as discussed in previous literature 18 The rest of the notation is standard, including a superexchange term with coupling $J_{\mathrm{AF}}$, which can be made spatially inhomogeneous due to the introduction of quenched disorder in the form of a bimodal distribution of amplitude $\Delta$ (that will always be expressed in units of $t_{a a}^{x}$ ), namely at each link the local coupling $J_{\mathrm{AF}}(i, \alpha)$ is either $J_{\mathrm{AF}}+\Delta$ or $J_{\mathrm{AF}}-\Delta$ with equal chance. This type of disorder is natural if the effect of chemical doping in manganites is considered, where ions of a given size are replaced by ions of a 
different size creating local stress in the crystal. Also, in some of the investigations reported here, a Zeeman term with field strength $B$ was added to investigate the influence of magnetic fields.

The two orbital model was here studied mainly using the canonical Monte Carlo method for spin-fermion systems, widely described in previous literature $\underline{\underline{5}}$ The conductances were calculated using a method proposed by Verges 27 and the unit of conductance throughout this paper is $e^{2} / h$. The lattices studied here are of intermediate size (with the exception of those in Section VI using the TPEM), but note that the computational effort was substantial (see below) in view of the many parameters in the problem, plus the need of averaging over several configurations of disorder. Future work exclusively focused on a small region of parameter space could address even larger systems.

It is also important to remark that the computational work described in this manuscript has several unique characteristics, including the vast resources that it has required to accurately simulate the manganite models. To give the reader a perspective of the effort, note that most of the simulations have been carried out on the NCCS XT3 supercomputer (with 2.4-GHz AMD Opteron processors and 2 GB of memory), using 100 nodes in parallel for small systems and up to 1600 nodes per run on large lattices. The entire effort documented in this paper would have required several years using a cluster of commodity PCs. Parallelization has been implemented with the MPI at three levels. First, the integration of the electronic sector, which is carried out with the TPEM, has been parallelized. The next level is related to the presence of quenched disorder in the system that requires the use of many different disorder configurations. Finally, the simulations have to sweep over a large parameter space $\left(\lambda, J_{\mathrm{AF}}\right.$ and temperature) allowing for a third level of parallelization.

\section{EFFECT OF LINK DISORDER ON THE RESISTANCE}

In this section, the effect of quenched disorder (in the $J_{\mathrm{AF}}$ link variables) on the insulating phases that appear in models for manganites will be investigated. Our main goal is to analyze if these insulators are robust, namely if they preserve their properties with increasing disorder, or whether they are fragile under the same "perturbation". Surprisingly, the latter is found to be the case: large changes in the properties of the insulating state occur even for fairly modest disorder strengths. This is in good agreement with several experiments in the manganite context, as explained before. Our results provide evidence that some states, such as the $\mathrm{CE}$, are fragile, namely easily disturbed by perturbations. The study in this section is organized following the sketch given in Fig. 1for a 3D case. First, the A-type antiferromagnetic state will be studied, followed by the CE phase. These

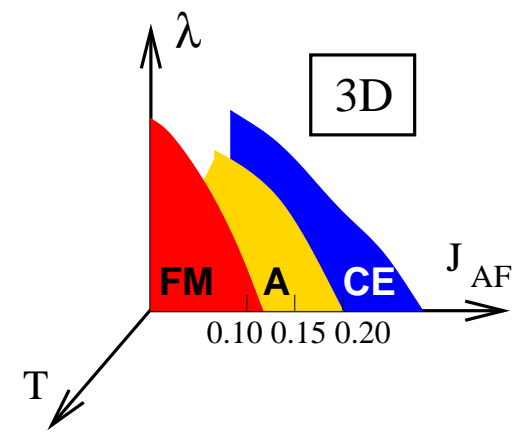

FIG. 1: Schematic representation of the phase diagram corresponding to the model Eq. (11) in three dimensions, ${ }^{40}$ and electronic density $n=0.5$. The influence of quenched disorder on the conductance will be mainly studied in this case in the $\lambda=0$ plane ( $i$. e.without electron-phonon coupling), focusing on the A-type and CE phases.

are the closest states to the important FM metallic state. At the end of the section, the 2D CE state is also analyzed.

\section{A. A-type antiferromagnet in 3D}

The A-type AF phase consists of ferromagnetically ordered planes, that are antiferromagnetically stacked along one axis. At $\lambda=0$ and in $3 \mathrm{D}$, this state is stable at intermediate values of $J_{\mathrm{AF}}$, as shown in Ref. 40 . It is interesting to observe that this phase is obtained in our study using unbiased Monte Carlo simulations 5.41 in the clean limit $(\Delta=0)$ starting, for instance, from a fully paramagnetic (random) classical spin configuration. There are no problems identified here of convergences to higher energy states. However, note that due to its intrinsic anisotropy, the conductance of the A-type AF phase is highly directional, $i$. e., the system is metallic in the FM plane but insulating when the conductance is measured along the direction where the system is AF. In Monte Carlo simulations, while the A-type character of the state is always obtained in the appropriate range of parameters, the orientation (i. e.the antiferromagnetic direction) is random. This is clear since the three orientations are energetically degenerate. Since our goal is to study the A-type AF insulator, as it appears in experiments where it has an insulating character, then we measure the conductance along the insulating direction of the A-type phase.

In Fig. 2 the conductance vs. temperature, $T$, is plotted for various disorder strengths, $\Delta$. The study is carried out at $J_{\mathrm{AF}}=0.15$, which we found corresponds to an A-type antiferromagnetic ground state for $\Delta=0$. Shown are averages over 5 configurations of quenched disorder. At $\Delta=0$, the conductance vanishes at low temperatures, result compatible with the expected insulating properties of the A-type AF phase (the spin-spin correlations and spin structure factor indeed confirm the A-type spin 


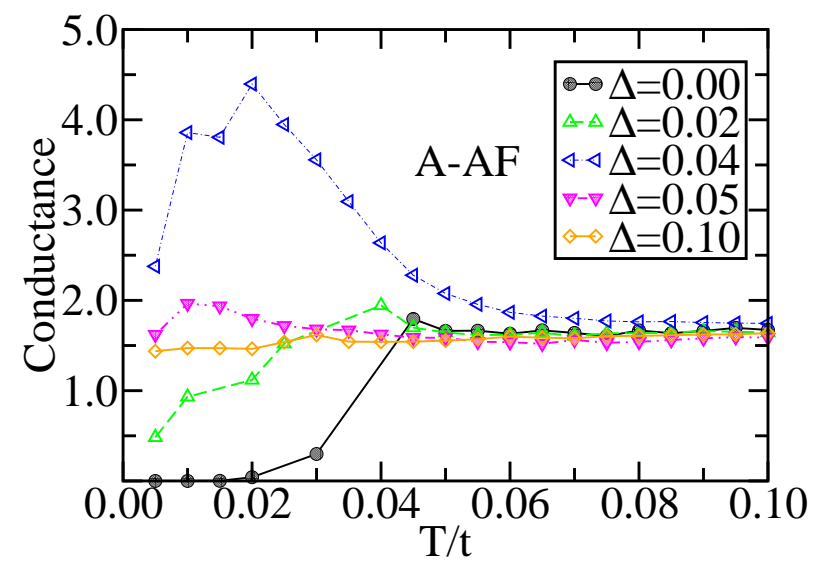

FIG. 2: Results illustrating the appearance of a metal upon adding quenched disorder to an insulator. Shown is the numerically calculated conductance vs. temperature $T$ for the A-type AF phase in three dimensions, with the antiferromagnetic spin arrangement along the $z$-axis, with and without disorder. The calculation was done on a $4^{3}$ lattice, working at $J_{\mathrm{AF}}=0.15$ and $\lambda=0$. For each temperature and $\Delta$, 5,000 thermalization and 15,000 measurement Monte Carlo steps through the whole lattice were carried out. The actual measurements of observables were separated by 10 steps. The starting configuration corresponded to an A-type AF in the $z$-direction. The link disorder is bimodal, $J_{\mathrm{AF}} \pm \Delta$, and the average shown is over 5 quenched disorder configurations. For the error related to this average see Fig. [3].

character of this state).

The most interesting results of Fig. 22 are obtained at nonzero $\Delta$. Results at $\Delta=0.02$ indicate an enhancement of metallicity (higher conductance) than in the clean $\Delta=0$ limit, which is surprising. The system has a finite conductance in this regime. At $\Delta=0.04$, (a disorder strength that can be considered intermediate with respect to the central value of the disorder distribution, $\left.J_{\mathrm{AF}}=0.15\right)$, the effect reported in this paper is the most dramatic. At this value of $\Delta$, the low temperature conductance reaches a maximum. Upon further increasing $\Delta$, the transport properties now become that of a poor metal, namely the actual conductance value is low (at least compared with its maximum possible value), and the result is nearly temperature independent. It is the surprising regime of small/intermediate $\Delta$ that it is the most interesting due to its dramatic change in conductance, but the large $\Delta$ region is also puzzling since at very large disorder one would expect to find an insulator.

In studies incorporating quenched disorder, the averages over disorder configurations are important. Indeed, disorder introduces another source of error (apart from Monte Carlo error) and the size of this error needs to be determined. To that effect the dependence of the conductance on each of the five different disorder configurations used in the previous figure is shown in Fig. B $\mathrm{k}$ for $\Delta=0.1$. We found an analogous behavior for other values of $\Delta$ although this error tends to be larger for smaller $\Delta \mathrm{s}$. Moreover, a similar dependence of the conductance
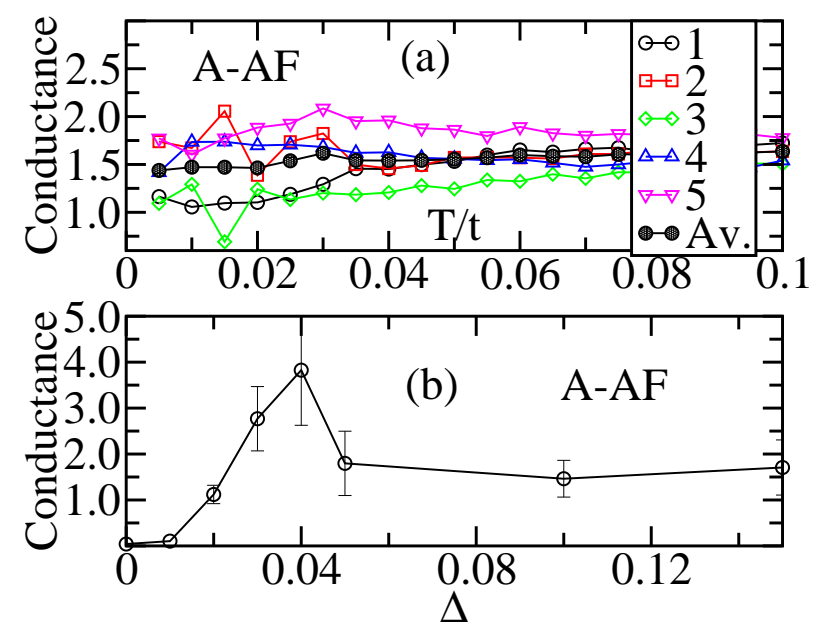

FIG. 3: (a) Dependence of the conductance on the particular quenched-disorder configuration used (labeled as 1-5, with "Av." denoting the average). Although the results near the metal-insulator transition vary substantially from configuration to configuration, the qualitative behavior is similar in all of them. Shown is the conductance vs. $T$ for the A-type $\mathrm{AF}$ phase (AF along the $z$-axis) for different disorder configurations, using $\Delta=0.1$. The calculation was done on a $4^{3}$ lattice at $J_{\mathrm{AF}}=0.15$ and $\lambda=0$, with the same number of Monte Carlo steps discussed in Fig 2 The disorder is bimodal, $J_{\mathrm{AF}} \pm \Delta$, and the average shown is over the 5 disorder configurations. (b) Dependence of the conductance on the disorder strength $\Delta$, at low temperatures, showing the transition from an insulator to a metal upon disordering the system. Shown is the conductance vs. $\Delta$ of the A-type AF phase (AF along the $z$-axis) at $T=0.02 t$. The lattice, couplings, Monte Carlo steps, initial configuration, and type of disorder used are as in (a). The error bars mainly arise from the average over several quenched disorder configurations.

on the disorder configuration was found for the CE phase.

The low temperature $(T=0.02)$ dependence of the conductance on the disorder strength, $\Delta$, is shown in Fig. 3 b. It is remarkable that the connection between the insulating state at $\Delta=0$ and the large $\Delta$ limit is not smooth, but a peak is observed at intermediate disorder strength.

A possible explanation of the results obtained in this study is the following. Let us start at very large $\Delta$, namely in the regime where disorder dominates. It may appear unnatural that in such a limit a metal (even if poor) is obtained, since disorder is associated with localization and related effects. However, in the large $\Delta$ regime each of the lattice links (defined by two nearest neighbor sites) has an associated pair of classical spins which are either mainly ferromagnetic or antiferromagnetically aligned, depending on the value of the coupling $J_{\mathrm{AF}}$ at that particular link. If the link coupling is $J_{\mathrm{AF}}+\Delta$, then this link is AF, while for $J_{\mathrm{AF}}-\Delta$ it tends to be ferromagnetic due to the double exchange mechanism. The fact that at large $\Delta$ the orientation of the classical spins at each link follows the disorder is clear from Fig团(top panels) where both the spin correlations 
and the $J_{\mathrm{AF}}$ couplings are shown. Although a few links have different color (or thickness) on the left and right top panels, the majority coincide, namely the spins follow the couplings. The important point in this regime is that the ferromagnetic links can percolate since there are (on average) $50 \%$ of them, and in three dimensions the critical concentration for percolation is only $25 \%$. A percolative path is shown in the lower panel of Fig प However, note that this explanation is only rough, since for Heisenberg classical variables (i. e.defined by two angles) as in our simulations the correlation between two links does not necessarily take two values (FM/AF) as in the case of Ising spins. Nevertheless, we have observed that even within the many percolative channels, the spins tend to twist their orientations to satisfy better the competing FM-AF tendencies reducing the conductance substantially. The situation would be different in systems with sufficient anisotropy to be described by Ising variables. The important fact is that even if the FM links are percolated, the AF links provide a frustrating tendency to them, $i$. e.they are not independent of each other. Thus, the resulting conductance is finite but poor. Nevertheless, the essence of the metallic behavior found at large $\Delta$ is related to the percolative picture described in this paragraph.

For low and intermediate disorder strengths, the conductance is larger than at large $\Delta$. A different, more complex explanation of the results holds in this regime, and this will be discussed later in this paper, after confirming that the large intermediate conductance occurs in other systems as well.

\section{B. CE phase in $3 \mathrm{D}$}

An investigation similar to that reported in the previous section for the A-type $\mathrm{AF}$ phase was also carried out for the CE phase. The CE state is stabilized in the clean limit by simply further increasing $J_{\mathrm{AF}}$ with respect to the value used for the A-type AF states, as reported in previous investigations (see sketch Fig प). In Fig. 国(a) the conductance vs. $T$ is presented for $J_{\mathrm{AF}}=0.2$ (corresponding to a CE-phase ground state at $\Delta=0$ ), and for various strengths of the disorder configuration. At $\Delta=0$, the expected result is found, namely for temperatures below a critical one the conductance vanishes. This clearly signals an insulator, which has CE characteristics based on the values of the spin and charge correlations $\frac{13}{\underline{13}}$ For small values of $\Delta$, the conductance increases from zero at low temperatures, and at $\Delta=0.10$ it reaches a plateau, which is nearly temperature independent. Note that contrary to the results for the A-type AF phase, the conductance grows monotonically until the large $\Delta$ (percolated) regime is reached. It will be argued later that this is caused by the fact that the FM metallic phase is no longer a neighbor of the $\mathrm{CE}$ state in the 3D phase diagram. For completeness, the case of a FM ground state (stabilized at smaller values of $J_{\mathrm{AF}}$ ) is also shown in Fig. 囵(b). At

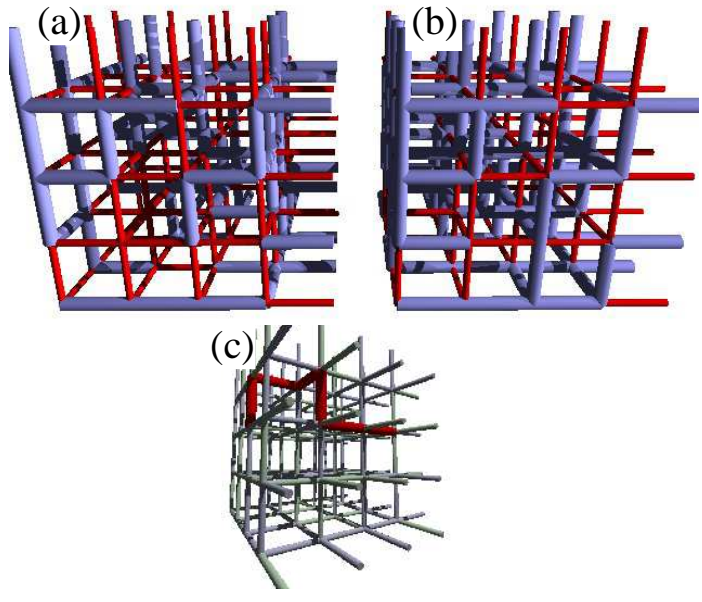

FIG. 4: Figures illustrating the expected dominance of quenched disorder over the spin configurations, at large $\Delta$. (a) Sign of $\vec{S}_{i} \cdot \vec{S}_{j}$ for nearest-neighbor sites $i, j$, indicated as red/thin (negative sign; antiferromagnetic) and blue/thick (positive sign; ferromagnetic) connections. The result corresponds to one particular quenched disorder and classical spin configurations, obtained after the Monte Carlo thermalization. (b) Value of the link coupling $J_{\mathrm{AF}}(i, j)$, indicated as colors red/thin $\left(J_{\mathrm{AF}}+\Delta\right)$ and blue/thick $\left(J_{\mathrm{AF}}-\Delta\right)$. In both left and right panels, the couplings were $J_{\mathrm{AF}}=0.15$ and $\Delta=0.1$. The correlation between the results in both panels is apparent, indicating that for this large value of the disorder strength the classical spins follow the disorder in the spin-spin correlations. (a) An example of a FM percolative path found in the study of the top panels. There are many of them (but only one shown).

all temperatures, the conductance decreases with increasing disorder strength. For large enough $\Delta$ (not shown), a common limit is reached both in the cases of the (a) and (b) panels in the figure. The metallic FM state is damaged by disorder and its conductance is reduced, as intuition suggests, while the insulating states (A or $\mathrm{CE}$ type) actually become metallic after the addition of disorder.

\section{CE phase in $2 \mathrm{D}$}

To verify the universality of the rapid transformation of the $\mathrm{CE}$ phase into a poor metal upon introduction of disorder found in 3D, similar calculations have been carried out in 2D. In this case, a plethora of previous investigations ${ }^{-5}$ have shown that the CE phase is stabilized at $n=0.5$ by increasing the coupling $J_{\mathrm{AF}}$. A typical phase diagram is sketched in Fig. [6 Note that the CE phase is stabilized even at $\lambda=0$, mainly for topological reasons related with the kinetic energy of carriers in zigzag chains ${ }^{25}$ However, in the analysis below, we will also study the CE phase in the regime where the electronphonon coupling $\lambda$ is important, to search for qualitative differences. 


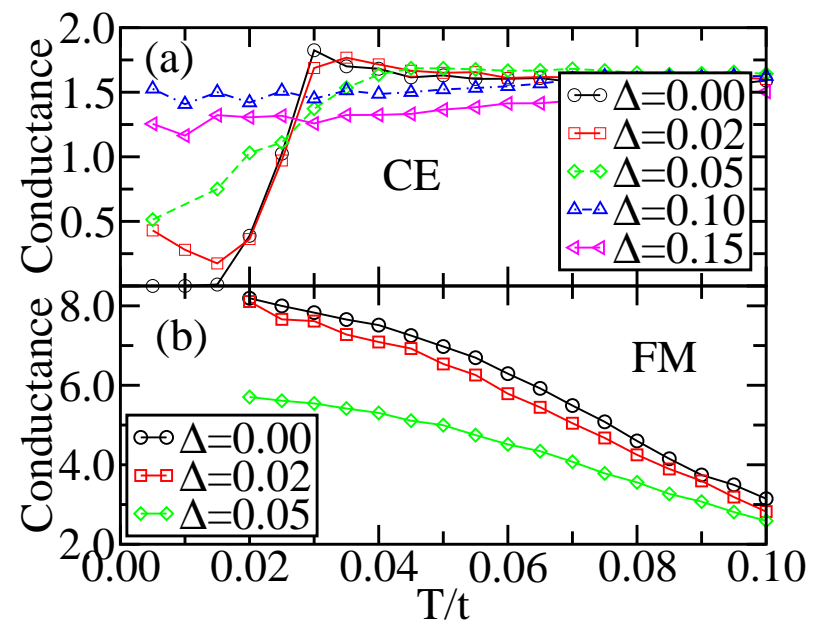

FIG. 5: (a) Results illustrating the metal to insulator transition induced by disorder, focusing on the regime of the $\mathrm{CE}$ phase in a three-dimensional lattice. Shown are the conductance vs. $T$ of the CE-phase, with and without quenched disorder of strength $\Delta$. The calculation was done on a $4^{3}$ lattice, at $J_{\mathrm{AF}}=0.20, \lambda=0$, and using 5,000 for thermalization and 15,000 for measurement Monte Carlo steps (the latter actually evaluating observables at every 10 steps to avoid self-correlations). The initial starting configuration had a CE phase with zigzag chains in the xy-plane. The disorder is bimodal, $J_{\mathrm{AF}} \pm \Delta$, and shown are averages over 5 disorder configurations. (b) Conductance vs. $T$ for the FM phase, with and without disorder. In this case, where a metal exists at $\Delta=0$, the disorder simply decreases the conductance, as intuitively expected. The lattice, Monte Carlo steps, number of disorder configurations, and type of link disorder are as in (a). The superexchange coupling is $J_{\mathrm{AF}}=0.10$.

Figure 7(a) shows the effect of disorder on the CE phase for various strengths $\Delta$ and with $\lambda=0$. The results present interesting similarities with those obtained for the A-type AF phase in 3D: a conducting system is rapidly stabilized by increasing $\Delta$ and this phenomenon occurs in a non-monotonic way, with fairly small $\Delta$ 's having the largest effect in the conductance. At the lowest temperatures the system has an insulating behavior that becomes metallic at intermediate temperatures and finally it achieves constant conductance at the high temperature paramagnetic regime ${ }^{42}$ It is interesting to observe that increasing $\lambda$, the rapid transformation of the insulator into a metal no longer occurs, as shown in Fig. 7(b) for the case $\lambda=1$. While a nonzero conductance is reached at low temperatures, its value is substantially smaller than in the $\lambda=0$ case. This effect is more pronounced in Fig. 8]a), where the conductance at low temperatures $T=0.01$ is shown. The large difference between the small and large $\lambda$ regimes is obvious: at small $\lambda$ the fragility of the CE state is much more notorious. Also note the similarity of the conductance vs. $\Delta$ plot for the 2D CE state with the results for the 3D A-type AF state. We believe the reason is that both these states are the closest to the FM metallic state in parameter space,

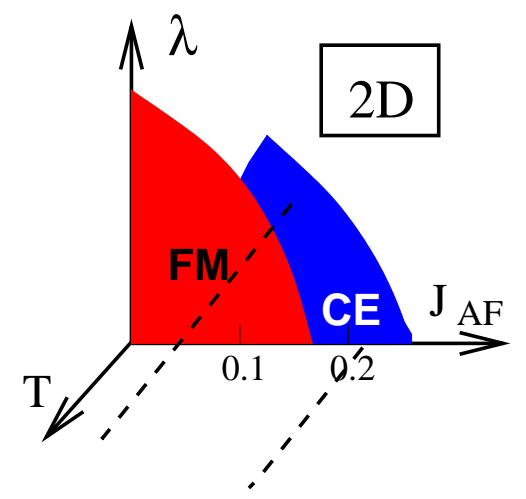

FIG. 6: Schematic phase diagram for model Eq. (1) in two dimensions, from Ref 18 . The effect of quenched disorder is studied in this section in the cases: (i) $\lambda=0, J_{\mathrm{AF}}=0.17$, corresponding to a $\mathrm{CE}$ phase without active phonons, and (ii) $\lambda=1, J_{\mathrm{AF}}=0.145$, corresponding to a CE phase stabilized including phonons. These two cases are indicated by dashed lines.

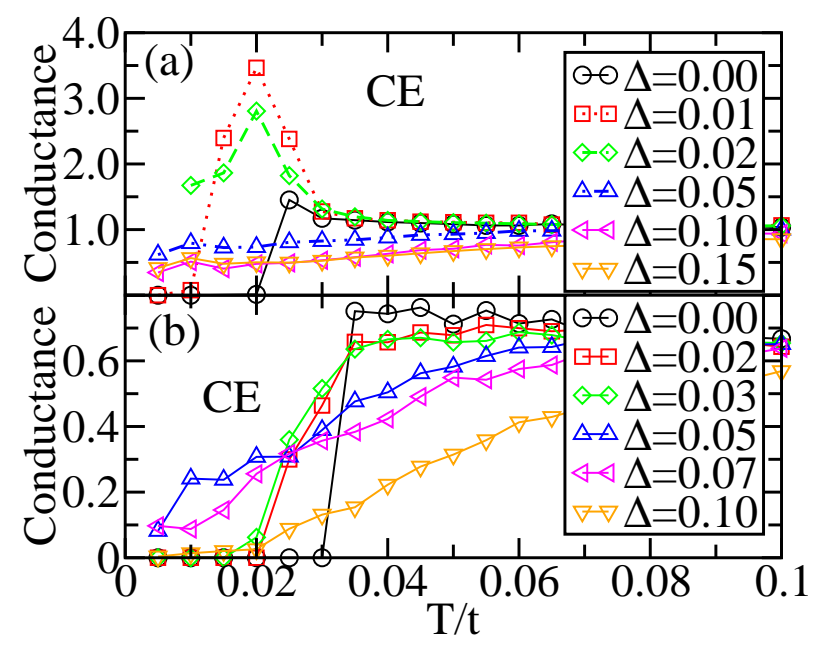

FIG. 7: Insulator to metal transition induced by quenched disorder in two dimensions. (a) Shown is the conductance vs. $T$ of the two-dimensional CE phase, for different disorder strengths $\Delta$. The calculation was done on an $8 \times 8$ lattice, at $J_{\mathrm{AF}}=0.17$, and $\lambda=0$. The number of Monte Carlo steps was 5,000 for thermalization and 15,000 for measurements, calculating observables every 10 steps. The initial configuration was the perfect $\mathrm{CE}$ phase. The disorder is bimodal, $J_{\mathrm{AF}} \pm \Delta$, and the averages shown are over 5 quenched disorder configurations. (b) Same as (a) but for $J_{\mathrm{AF}}=0.145$ and $\lambda=1.0$.

as explained in more detail below.

Finally, the clear correlation between the spin orientations 48 at each link and the value of $J_{\mathrm{AF}}$ at that particular link is shown in Fig. 8(b,c) at large $\Delta$. As in $3 \mathrm{D}$, percolative processes favor a poor metallic behavior in this regime. But note that $50 \%$ is the critical number of links for 2D percolation and, as a consequence, the 2D large $\Delta$ regime is only at the verge of metallicity. This explains the smaller values in conductance at large 

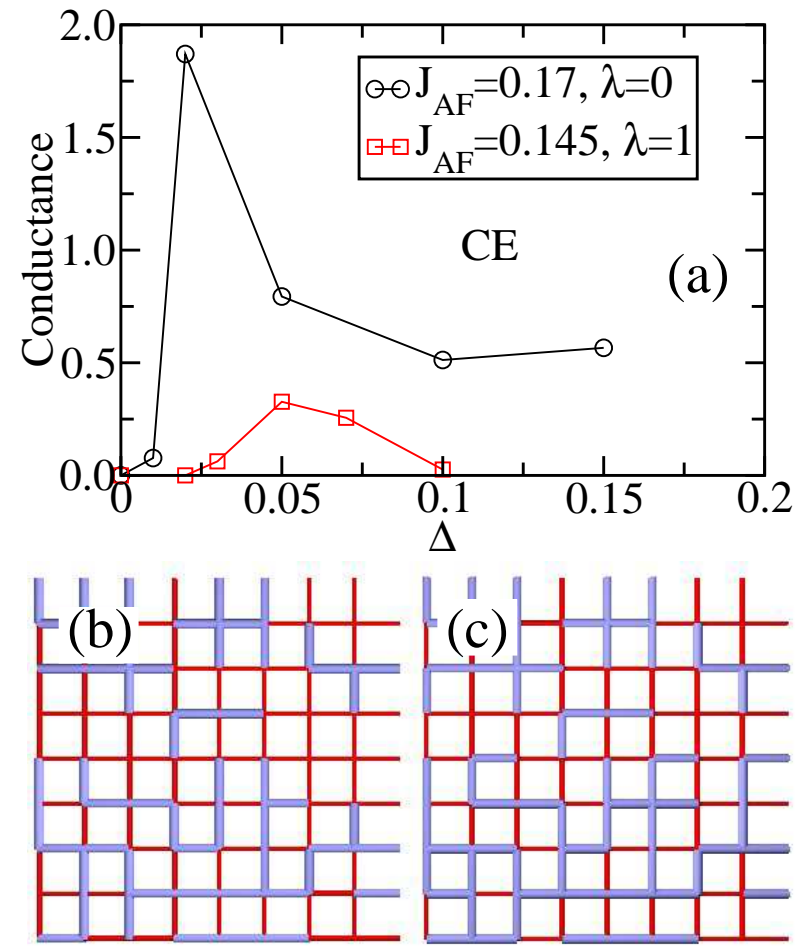

FIG. 8: (a) Dependence of the conductance on disorder strength $\Delta$, at low temperature $T=0.01$, using results taken from Fig. 17a). The most dramatic evidence of fragility in the 2D insulating CE state is obtained in the absence of electronphonon coupling. (b) Sign of $\vec{S}_{i} \cdot \vec{S}_{j}$ for nearest-neighbor sites $i, j$, indicated as red/thin (negative; AF) and blue/thick (positive; FM) connections. (c) Value of $J_{\mathrm{AF}}(i, j)$ indicated as two colors red/thin $\left(J_{\mathrm{AF}}+\Delta\right)$ and blue/thick $\left(J_{\mathrm{AF}}-\Delta\right)$. In both cases, $J_{\mathrm{AF}}=0.17, \Delta=0.1$, and the results were obtained for fixed classical spin and quenched disorder configurations. The correlation between the quantities in the (b) and (c) panels is apparent, indicating that for this large value of $\Delta$ the system follows the disorder in the spin-spin correlations.

$\Delta$ between $2 \mathrm{D}$ and $3 \mathrm{D}$. The state induced by the disorder acting on the $2 \mathrm{D}$ CE state must be similar to the "CE glass" studied in Ref. 18 before.

\section{EFFECT OF DISORDER ON THE MAGNETO-RESISTANCE}

The fragility of the insulating states in the $\lambda=0$ regime upon the introduction of disorder, suggests that other perturbations may also rapidly transform these states from insulating to metal. In particular, the effect of magnetic fields, $B$, is important for potential applications and to improve our understanding of the CMR effect of manganites. To carry out this study, we will proceed sequentially through the phases and parameters described before and summarized in Figs. 1 and 6 The magnetoresistance here will be defined by $[(R(0)-R(B)) / R(0)] \times$ 100 , where $R(B)$ is the resistance (1/conductance) at the magnetic field $B$. The magnetic field is applied in the $z$ direction, same orientation as the $\mathrm{A}$ and $\mathrm{CE}$ phases, and we have chosen the intensities of $B=0.05$ and $B=0.10$ for our studies.

\section{A. A-type AF phase}

Results for the conductance in the 3D A-type AF regime are given in Fig. 9] in the clean and disordered cases, and considering both $B=0$ and $B=0.1$. It is remarkable that in the clean limit, a relatively small field such as $B=0.1$ is capable of totally transforming the insulator into a metal. In this case, the magneto-resistance factor is the largest, very close to $100 \%$, as shown in Fig. 10] A similar phenomenon occurs at $B=0.05$ (not shown). The magnitude of this effect remains strong for small $\Delta \mathrm{s}$ such as 0.02 , and then slowly decreases. The singular behavior at temperatures close to $T=0.03-0.04$ is caused by the peaks in conductance at those intermediate temperatures. The focus should be on the low temperature regime, which is the most interesting in view of the magnitude of the effects. The result at $\Delta=0$ is probably caused by the close proximity in energy of the FM metallic state, as discussed before in Ref.18. If this is the case, then the transition must be first-order between the two, and a finite value of $B$ is needed to induce it. From this perspective, the finite but small $\Delta$ region will be more interesting since even in the limit of vanishing $B$, it has a substantial magnetic susceptibility, as discussed below.

\section{B. CE-phase in $3 \mathrm{D}$ and $2 \mathrm{D}$}

Monte Carlo simulations with an applied magnetic field were also carried out in the regime of the CE phase in three dimensions, and the results are shown in Fig. 11 for $B=0.1$. As in the previous case of the A-type phase, here also the CE phase is found to be highly susceptible to the application of a magnetic field, and the clean limit insulating state quickly becomes metallic. Disordered samples are less susceptible to the application of a magnetic field, as previously observed for the A-type $\mathrm{AF}$ phase. Both regimes, $\mathrm{CE}$ and A-type $\mathrm{AF}$, present a similar phenomenology.

The analysis of the influence of magnetic fields can be made more precise by studying the magnetization at small fields $B$. The results are shown in Fig. 12 At $\Delta=0$, a small but finite $B$ is needed to render the system ferromagnetic, as previously discussed. This is probably related with the first-order transition reported in previous investigations in the clean limit 18 The slope of $M$ vs. $B$ at low fields increases with $\Delta$. This small $\Delta$ regime is also the most relevant in some of the cases studied in previous sections in the absence of magnetic fields.

To further investigate the origin of these effects, Monte Carlo thermalized spin configurations have been analyzed. From each of these configurations we constructed 


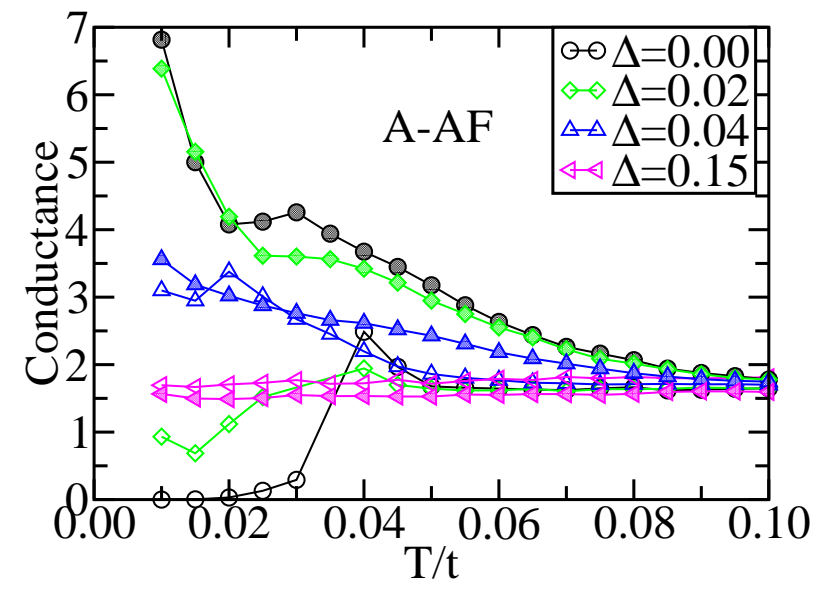

FIG. 9: Influence of a magnetic field on the transport properties of the 3D A-type AF state, as discussed in the text. The effect is particularly dramatic at small $\Delta$, including the clean limit $\Delta=0$. Shown are the conductances vs. $T$ of the Atype AF phase, at different disorder strengths $\Delta$. Filled symbols (empty symbols) correspond to a magnetic field $B=0.1$ $(B=0.0)$. The calculations were done on a $4^{3}$ lattice, at $J_{\mathrm{AF}}=0.15$ and $\lambda=0$, and using 5,000 thermalization and 15,000 measurement Monte Carlo steps (calculating the conductance every 10 steps). The starting configuration used was an A-type AF phase. The disorder is bimodal, $J_{\mathrm{AF}} \pm \Delta$, and shown are averages over 5 disorder configurations. The magnetic field is applied in the $z$-direction.

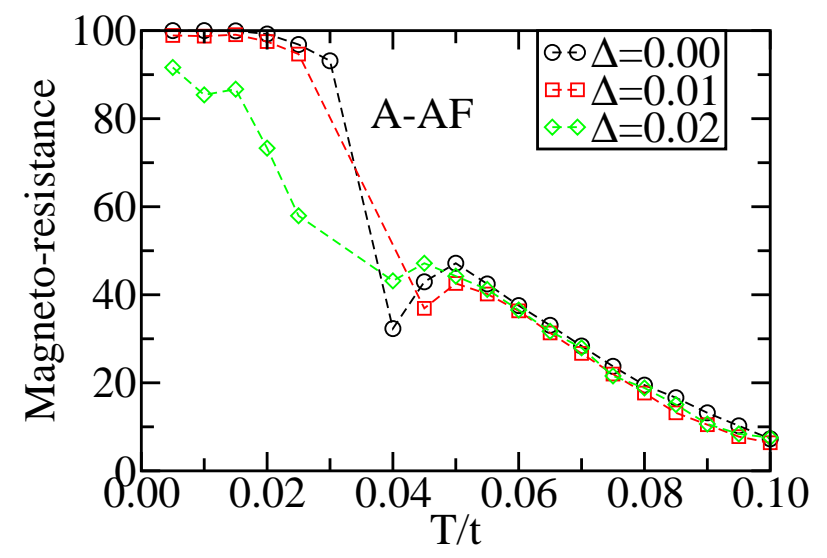

FIG. 10: Magneto-resistance (defined in Section IV] at $B=0.1$ vs. $T$, for the A-type $\mathrm{AF}$ regime in $3 \mathrm{D}$, and considering several disorder strengths $\Delta$ (indicated). The lattice, couplings, Monte Carlo steps, and other conventions are as in Fig. [9]

the "summed snapshot", $\sum_{j \in \mathcal{V}_{i}} \vec{S}_{j}$, where $\mathcal{V}_{i}$ represents the vicinity ( 4 sites in $2 \mathrm{D}$ or 6 sites in 3D) of site $i$. The advantage of this quantity is that it helps identify regions that are FM. If the spins near $i$ are pointing randomly then the "summed" spin tends to cancel. In Fig. 13](a), an example is shown using an $8 \times 8$ lattice (very similar results were obtained on a $20 \times 20$ cluster using the TPEM method described later in this paper). In the absence of magnetic fields, but including disorder with a

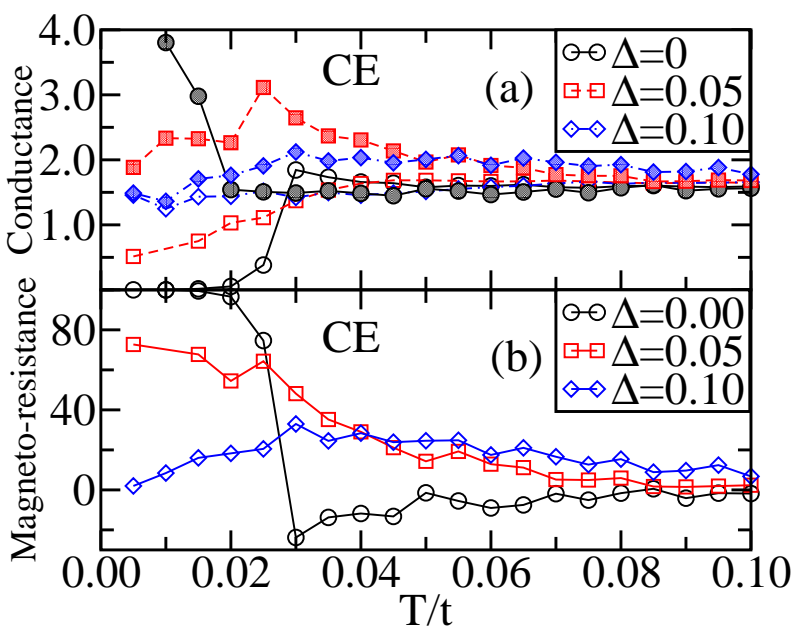

FIG. 11: (a) Influence of a magnetic field on the transport properties in the regime of the CE phase in three dimensions. Shown are the conductances vs. $T$ of the CE-type phase, for different disorder strengths $\Delta$. Filled symbols (empty symbols) correspond to magnetic fields $B=0.1 \quad(B=0.0)$. Note the large change at low temperatures, and small and vanishing disorder strength. Lattice, $\lambda$, Monte Carlo steps, number of quenched disorder configurations for averages, and other details are as in Fig[9] The coupling $J_{\mathrm{AF}}$ is 0.2. (b) Magnetoresistance (defined in Section IV) corresponding to the data shown in (a).

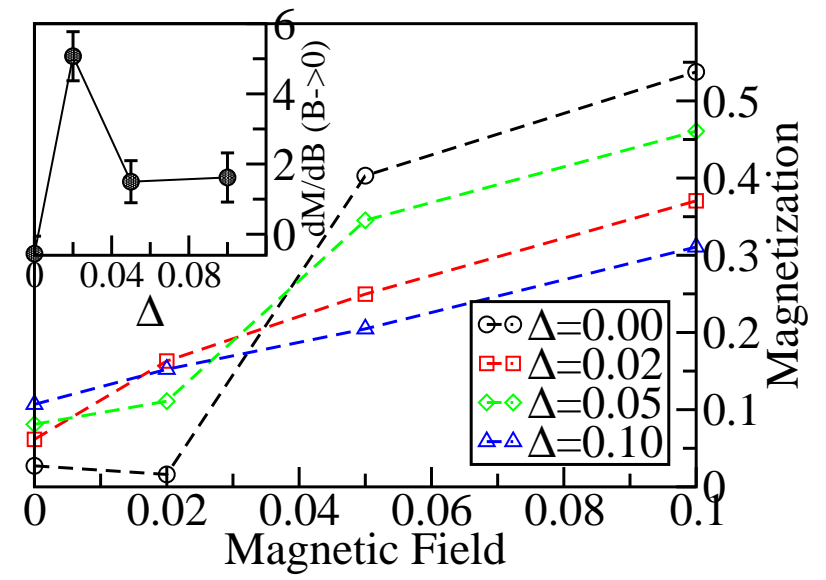

FIG. 12: Magnetization vs. applied magnetic field, for various disorder strengths $\Delta$ at $T=0.01$. Inset: The magnetic susceptibility $d M /\left.d B\right|_{B \rightarrow 0}$ vs. disorder strength $\Delta$. Parameters and other details are as in Fig. 11]

small $\Delta$, the stabilized spin configuration is very different from the CE phase with parallel zigzag chains that is present at $\Delta=0$. With this disorder, ferromagnetic clusters containing many spins with the same orientation are present. Their random orientation character gives a nearly zero global magnetization. However, clearly a system with preformed FM islands must have a high susceptibility to external magnetic fields, as already argued to be the reason for the manganite CMR effect in previ- 

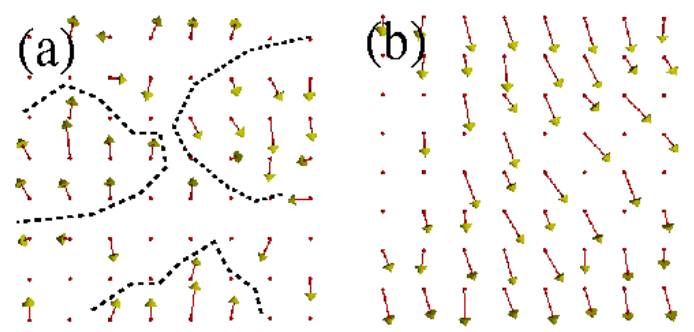

FIG. 13: (a) Monte Carlo "summed snapshot" showing the "clustered spins" stabilized by a small amount of quenched disorder at $B=0$, for the case of an $8 \times 8$ lattice, $J_{\mathrm{AF}}=0.17$, $\lambda=0$, and $\Delta=0.02$. In (a), the presence of FM clusters is clear to the eye: quenched disorder with a small value of $\Delta$ transforms the original $\mathrm{CE}$ state into a state with local ferromagnetism (but not global). The conductance of this state is zero. (b) Same as in (a) but for $B=0.02$. A very small magnetic field is sufficient to align the pre-formed FM clusters, and simultaneously increasing the conductance (to a value $C=3$, in this case).

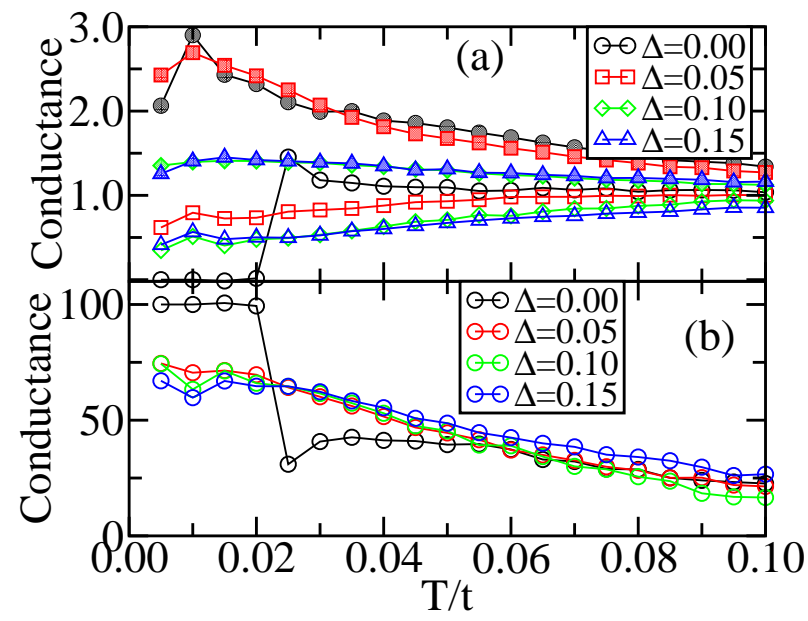

FIG. 14: (a) Conductance vs. $T$ of the two-dimensional CE phase for different disorder strengths $\Delta$. Full (empty) symbols correspond to a magnetic field $B=0.1(B=0)$. The calculation was done on an $8 \times 8$ lattice at $J_{\mathrm{AF}}=0.17, \lambda=0$, and using 5,000 thermalization and 15,000 measurement Monte Carlo steps, and the CE phase as initial configuration . The disorder is bimodal, $J_{\mathrm{AF}} \pm \Delta$, and the average shown is over 5 quenched disorder configurations. (b) Magneto-resistance at $B=0.1$ vs. $T$ for the 2D CE phase, at different disorder strengths $\Delta$, as indicated. The calculation was done using the same lattice, couplings, and other details as in (a).

ous studies $\frac{5}{\underline{5}}$ When magnetic fields are applied, the FM clusters rapidly orient their moments creating a global FM state, as shown in Fig. [13(b).

For completeness, the effect of a magnetic field on the $\mathrm{CE}$ phase in two dimensions was also studied. Results for the conductance and the magnetoresistance are in Fig. 114 for $\lambda=0$. The trends are very similar to those found in three dimensions.

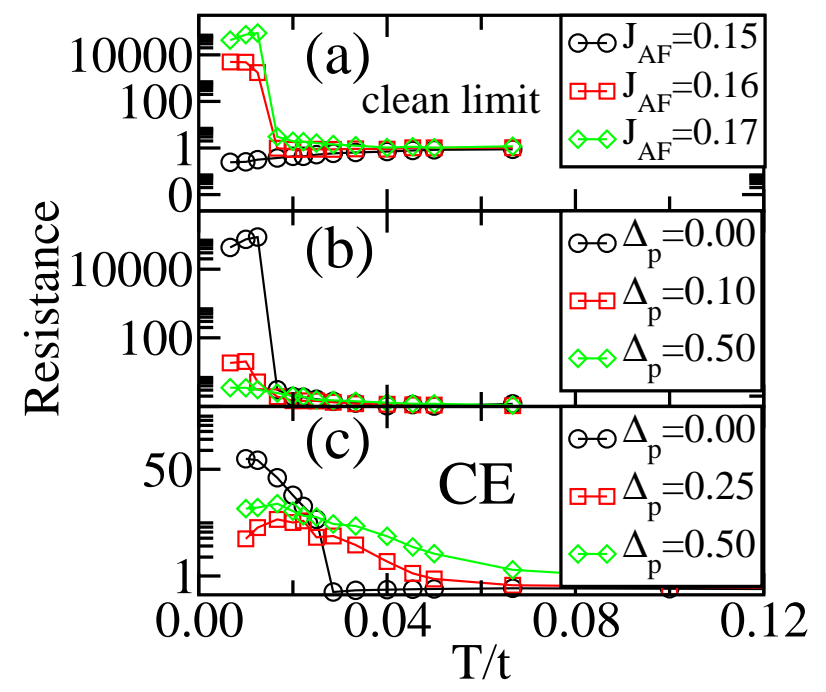

FIG. 15: (a) Resistance vs. temperature for a $12 \times 12$ lattice in the clean limit $\Delta=0, \lambda=0$, and considering $J_{\mathrm{AF}}=0.15$ (squares), $J_{\mathrm{AF}}=0.16$ (circles), and $J_{\mathrm{AF}}=0.17$ (triangles). The figure illustrates the metallic character of the $J_{\mathrm{AF}}=0.15$ state (with a FM ground state), and the insulating properties of the $J_{\mathrm{AF}}=0.16$ and 0.17 samples (with a $\mathrm{CE}$ ground state). The Monte Carlo simulation was carried out starting with a random state at each temperature, using a warming up of 10,000 steps, followed by an additional 10,000 steps to measure properties. (b) Same as (a) at $J_{\mathrm{AF}}=0.17$, but now considering the on-site disorder strengths $\Delta_{\mathrm{P}}$ indicated, and averaging over 3 different disorder configurations. The resistance of the clean sample is reduced several orders of magnitude by the addition of the on-site potential disorder, similarly as it occurs for link disorder. (c) Same as (a), but on a $4^{3}$ lattice and with $J_{\mathrm{AF}}=0.20$.

\section{RESULTS USING ON-SITE DISORDER}

In this section the influence of an on-site disorder (bimodal, of strength $\Delta_{\mathrm{P}}$ ) will be investigated. The term added to the Hamiltonian is an on-site random energy, $\sum_{i, \gamma} \epsilon_{i} c_{i, \gamma}^{\dagger} c_{i, \gamma}$ with $\epsilon_{i}= \pm \Delta_{\mathrm{P}}$, to be contrasted with the link randomness considered in the previous sections. It will be shown that the conclusions regarding the fragility of the insulating states at $\lambda=0$ remain valid, even if the source of disorder is changed.

Figure 15 a) shows the resistance obtained in the clean limit, parametric with $J_{\mathrm{AF}}$, using a $2 \mathrm{D}$ cluster. This result is the analog of the conductance plots in the clean limit presented in previous sections. Varying $J_{\mathrm{AF}}$, an abrupt transition from a metal to an insulator is obtained at low temperatures, as expected. Let us now introduce on-site disorder. The results are in Fig. 15 b). It is clear that the insulating state dramatically reduces its resistance with increasing $\Delta_{\mathrm{P}}$, transforming into a bad metallic state, as found in the case of link disorder. The changes in the resistance are of several orders of magnitude. The same occurs in three dimensions, Fig. 15I(c), where in addition it is shown that the resistance has a 


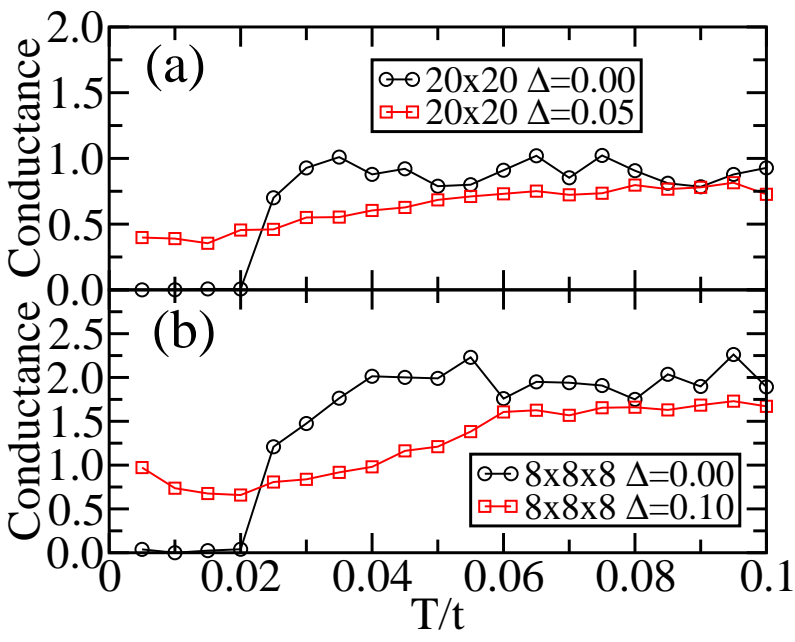

FIG. 16: (a) Effect of quenched disorder on the twodimensional $\mathrm{CE}$ phase, running the simulation with the TPEM. Conductance vs. $T$ of the CE phase on a $20 \times 20$ lattice calculated with the TPEM method using $M=30$ moments, and the TPEM parameters $\epsilon_{\mathrm{pr}}=10^{-6}$ and $\epsilon_{\mathrm{tr}}=10^{-6}$. The coupling was $J_{\mathrm{AF}}=0.17$, density $n=0.5$, and 2,000 for thermalization and 1,000 for measurement Monte Carlo steps were carried out. The CE phase shows zero conductance at low temperature in the clean limit. This transforms into a disordered metal with finite conductance when link quenched disorder of strength $\Delta=0.05$ is added. (b) Effect of disorder on the three-dimensional CE phase, studied with TPEM. Details are the same as in (a) but on an $8^{3}$ lattice and at $J_{\mathrm{AF}}=0.20$.

minimum at intermediate values of the disorder strength $\Delta_{\mathrm{P}}$, as it occurs for link disorder. Although the substantial numerical effort involved in the simulations presented in this manuscript prevents us from discussing the site disorder case in as much detailed as the case of link disorder, nevertheless it is safe to conclude that site disorder has a phenomenology similar to that of link disorder, in the sense that by increasing $\Delta_{\mathrm{P}}$ a conductor is obtained.

\section{FINITE-SIZE EFFECTS AND TPEM TECHNIQUE}

The investigations reported in the previous sections have been carried out using intermediate size clusters, at the verge of the sizes that can be handled with the exact diagonalization treatment of the fermionic sector. Fortunately, the systematic effects observed, and similarities of results between different quenched disorder configurations, dimensionality, and insulating phases strongly point toward a robust effect, and we are confident that the essence of the phenomenon has been unveiled. Nevertheless, only the study of larger clusters would convincingly rule out spurious size effects in our investigations. Fortunately, recent algorithm developments have led to the use of the "Truncated Polynomial Expansion Method" (TPEM) $43,44,45,46$ as an alternative to conven- tional Monte Carlo methods where the fermionic sector is treated exactly. The advantage of the TPEM is that its CPU time grows linearly with the number of sites $N$, while the canonical approach used in the previous sections demands an effort that scales as $N^{4}$. This advantage is not without penalization: the coefficient in front of $N$ in the TPEM scaling is very large. For this reason, we studied few large lattices, mainly to check if the results on small systems are preserved at larger $N$ values. After confirming that the TPEM reproduces the results of small systems accurately (not shown but see Ref. 47), studies were carried out using $20 \times 20$ and $8 \times 8 \times 8$ clusters. In Fig. 16 the results are shown, both in the clean limit and introducing disorder. In both cases, at low temperatures the conductance changes from exactly zero to a finite number, as observed in previous sections. While this is not a final proof, at least it has been confirmed that increasing the lattice sizes does not lead to dramatic changes in our conclusions, and the insulator to metal transition with increasing $\Delta$ seems to be a solid property of the CE states of manganites at small electron-phonon coupling.

\section{DISCUSSION AND CONCLUSIONS}

In this paper, the influence of small amounts of quenched disorder on the transport properties of manganite models was investigated. The focus has been on the insulating phases that are stabilized without electron-

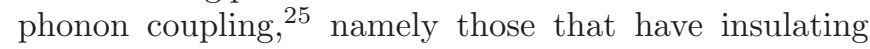
properties due to geometrical considerations and frustrating competing tendencies between FM and AF states.

The study reported here has identified regimes where the insulating phases, the $\mathrm{CE}$ and A-type AF states, are very susceptible to the introduction of disorder. For instance, the A-type $\mathrm{AF}$ phase in 3D and the CE-type phase in $2 \mathrm{D}$, they both present a conductance that sharply increases with increasing $\Delta$ from zero, and then it settles to a smaller value at large $\Delta$. The reason appears to be explained with the type of spin arrangements that become stabilized in the presence of quenched disorder. Typical results are shown in Fig. 17 for the case of the 2D CE state. In (a), the CE pattern is nicely reproduced by the Monte Carlo simulation at zero and very small $\Delta$. However, in (b), a key observation is that lines with classical spins align ferromagnetically are stabilized along the vertical direction for a small $\Delta$. For instance, the first and fourth column from the left are all FM. The second, fourth and fifth columns are almost entirely FM, with the exception of only one link. More quantitatively, there are 50 links FM in the vertical direction but only $27 \mathrm{FM}$ links in the horizontal direction (ratio approximately 2). These lines provide conducting paths for the charge, rendering the system conducting in the direction of those lines. Even though the conductance is not always measured along these lines, the average over all directions is still large. This is to be contrasted with 


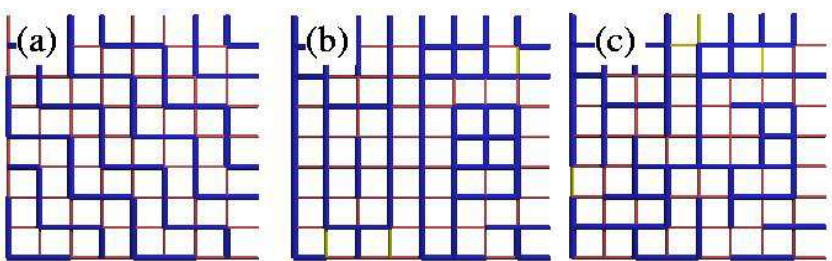

FIG. 17: Results of Monte Carlo simulations that summarize the main conclusions of this manuscript (see text for discussion). Shown are the signs of the classical spin correlation $\vec{S}_{i} \cdot \vec{S}_{j}$ considering nearest-neighbor sites $i, j$, indicated as red/thin (negative; AF) and blue/thick (positive; FM) connections. The lattice is $8 \times 8, J_{\mathrm{AF}}=0.17$, and the disorder strength is (a) $\Delta=0.01$, (b) $\Delta=0.02$, (c) $\Delta=0.08$. In (a), the disorder is too weak to change the CE spin pattern, which is clear in the result. However, in (b) the CE phase is replaced by long ferromagnetic (metallic) chains, which in this case are vertical. These chains provide conductance channels in the vertical direction. An equal number of horizontally conducting systems are found when analyzing many quenched disorder realizations, thus restoring rotational invariance after averaging. In (c), the state is more reminiscent of the high disorder regime, with shorter chains. At large $\Delta$, the spins follow the disorder as explained already in Section IIIC Of the three configurations, the largest conductance is obtained in (b).

the regime shown in (c) for large values of $\Delta$, where the ratio between FM links along the vertical and horizontal axes is approximately 1 , as is also in the clean limit. At least for $3 \mathrm{D}$, this type of configurations is also conducting, as explained with a percolative picture in previous sections. These tendencies were observed in numerous realizations of disorder, with the only difference between them being that the dominance of the horizontal or vertical lines has equal chance. Although most of the investigations reported here were obtained using intermediate size clusters, some larger systems were studied with the TPEM and the results remain similar. Nevertheless, a more sophisticated analysis is, of course, always desirable to confirm our conjectures. Hopefully, our effort will induce further work in this challenging area of research.

The origin of the FM lines that are rapidly stabilized by disorder must be the close proximity in energy of the full FM state. While the CE state prefers zigzag chains (which are band insulators ${ }^{25}$ ) to minimize the energy, the FM metallic state prefers straight lines since they are metallic. So this striped state can be considered as a compromise between the CE zigzag insulating and the FM uniform metallic states of the clean limit. Note that it is also possible to imagine a "clustered" state as a compromise of these two tendencies, and indeed in some circumstances this type of states was observed in our simulations, as it was shown in Fig. 13(a). But this state has zero conductance, while the striped state of Fig.17(b) has a finite conductance and, thus, it is responsible for the rapid insulator to metal transition with increasing $\Delta$.

We believe the conclusions reached in this paper have consequences beyond the case of Mn oxides. Clearly, the entire physics discussed arises from competition of phases, which is a common phenomenon in transition metal oxides. This phase competition is already present in the clean limit, since the CE and A-type AF phases appear in parameter space in narrow regions of the phase diagram, as a compromise between the FM and AF dominant tendencies. The same was recently shown to occur in between a $d$-wave superconductor and an antiferromagnet ${ }^{38}$ in a study of a phenomenological model for cuprates: in this case a striped phase was found as a compromise of the two tendencies in some regions of parameter space. It should not be surprising that the intermediate phases stabilized by phase competition are fragile, namely they change dramatically when influenced by disorder or external fields. This fragility manifests in the fairly exotic states that are stabilized when small quenched disorder is introduced: for the case of the manganites, these are states with either FM clusters or FM lines as reported here, having in common regions where ferromagnetism and metallicity dominates locally. That this occurs at values of $\Delta$ as small as 0.02 is the most surprising result. This high susceptibility to disorder should exist in many other complex oxides as well. When these giant effects are compared with other insulator to metal transitions induced by disorder (see Section \) it appears that the cases discussed here have a unique character, not previously discussed in the literature. The consequences of these results for our understanding of manganites and the CMR effect seem clear: it is the fragile nature of the insulator to the introduction of external perturbations (quenched disorder, magnetic fields, electric fields, ...) that may cause the well-known giant responses of $\mathrm{Mn}$ oxides.

\section{Acknowledgments}

Conversations with V. Dobrosavljevic are gratefully acknowledged. G. A. is supported by the Eugene P. Wigner Fellowship Program at Oak Ridge National Laboratory (ORNL). H. A., C. S. and E. D. are supported by the NSF grant DMR-0443144. H. A. is also supported by the LDRD program at ORNL. We acknowledge the National Center for Computational Sciences for providing most of the computational resources. ORNL is managed by UT-Battelle, LLC, for the U.S. Department of Energy under Contract DE-AC05-00OR22725.
1 Y. Tokura, Colossal Magnetoresistive Oxides (Gordon and Breach, New York, 2000).
2 E. Dagotto, Science 309, 258 (2005). 
${ }^{3}$ M. B. Salamon and M. Jaime, Rev. Mod. Phys. 73, 583 (2001).

4 M. Uehara, S. Mori, C. H. Chen, and S.-W. Cheong, Nature (London) 399, 560 (1999).

5 E. Dagotto, T. Hotta, and A. Moreo, Physics Reports 344, 1 (2001).

${ }^{6}$ N. Mathur and P. Littlewood, Physics Today January, 25 (2003).

7 C. N. R. Rao and B. Raveau, eds., Colossal Magnetoresistance, Charge Ordering, and Related Properties of Manganese Oxides (World Scientific, Singapore, 1998).

8 J. M. D. Teresa, M. R. Ibarra, P. A. Algarabel, C. Ritter, C. Marquina, J. Blasco, J. García, A. del Moral, and Z. Arnold, Nature 386, 256 (1997).

9 J. Lynn, R. W. Erwin, J. A. Borchers, Q. Huang, and A. Santoro, Phys. Rev. Lett. 76, 4046 (1996).

10 D. Khomskii and K. Kugel, Europhys. Lett. 55, 208 (2001).

11 A. Moreo, S. Yunoki, and E. Dagotto, Phys. Rev. Lett. 83, 2773 (1999).

12 K. H. Ahn, T. Lookman, and A. R. Bishop, Nature 428, 401 (2004).

13 E. Dagotto, ed., Nanoscale Phase Separation and Colossal Magnetoresistance (Springer Verlag, Berlin, 2002).

14 E. Dagotto, New Journal of Physics 7, 67 (2005).

15 S. Kumar and P. Majumdar, Phys. Rev. Lett. 90, 016602 (2006).

16 J. A. Verges, V. Martin-Mayor, and L. Brey, Phys. Rev. Lett. 88, 136401 (2002).

17 J. Salafranca and L. Brey (2006), cond-mat/0602392, Disorder-Induced First Order Transition and Curie Temperature Lowering in Ferromagnatic Manganites.

18 H. Aliaga, D. Magnoux, A. Moreo, D. Poilblanc, S. Yunoki, and E. Dagotto, Phys. Rev. B 68, 104405 (2003).

19 Y. Motome, N. Furukawa, and N. Nagaosa, Phys. Rev. Lett. 91, 167204 (2003).

${ }^{20}$ C. Sen, G. Alvarez, and E. Dagotto, Phys. Rev. B 70, 064428 (2004).

21 D. Akahoshi, M. Uchida, Y. Tomioka, T. Arima, Y. Matsui, and Y. Tokura, Phys. Rev. Lett. 90, 177203 (2003).

${ }^{22}$ T. Nakajima, H. Kageyama, and Y. Ueda, J. Phys. Chem. 63, 913 (2002).

23 J. Burgy, M. Mayr, V. Martin-Mayor, A. Moreo, and E. Dagotto, Phys. Rev. Lett. 87, 277202 (2001).

24 J. Burgy, A. Moreo, and E. Dagotto, Phys. Rev. Lett. 92, 097202 (2004).

25 T. Hotta, Y. Takada, H. Koizumi, and E. Dagotto, Phys. Rev. Lett. 84, 2477 (2000).

26 J. van den Brink, G. Khaliullin, and D. Khomskii, Phys.
Rev. Lett. 83, 5118 (1999).

27 J. A. Vergés, Comp. Phys. Commun. 118, 71 (1999).

28 P. Carpena, P. Bernaola-Galvan, P. C. Ivanov, and H. E. Stanley, Nature 418, 955 (2002).

29 P. Sheng, Phys. Rev. B 21, 2180 (1980).

30 E. J. Mele and M. J. Rice, Phys. Rev. B 23, 5397 (1981).

31 D. Tanasković, V. Dobrosavljević, E. Abrahams, and G. Kotliar, Phys. Rev. Lett. 91, 066603 (2003).

32 V. Dobrosavljević, D. Tanasković, and A. A. Pastor, Phys. Rev. Lett. 90, 016402 (2003).

33 J. Garcia, M. Sanchez, G. Subias, and J. Blasco, J. Phys.: Condens. Matter 13, 3229 (2001).

34 A. Daoud-Aladine, J. Rodriguez-Carvajal, L. PinsardGaudart, M. Fernandez-Diaz, and A. Revcolevschi, Phys. Rev. Lett. 89, 097205 (2002).

35 R. Mathieu, D. Akahoshi, A. Asamitsu, Y. Tomioka, and Y. Tokura, Phys. Rev. B 93, 227202 (2004).

36 J. M. Tranquada, B. J. Sternlieb, J. D. Axe, Y. Nakamura, and S. Uchida, Nature 375, 561 (1995).

37 S. A. Kivelson, I. P. Bindloss, E. Fradkin, V. Oganesyan, J. M. Tranquada, A. Kapitulnik, and C. Howald, Rev. Mod. Phys. 75, 1201 (2003).

38 G. Alvarez, M. Mayr, A. Moreo, and E. Dagotto, Phys. Rev. B 68, 014514 (2005).

39 M. Mayr, G. Alvarez, A. Moreo, and E. Dagotto, Phys. Rev. B 73, 014509 (2006).

40 H. Aliaga, Phys. Rev. B 71, 184404 (2005).

41 R. Kajimoto, H. Yoshizawa, Y. Tomioka, and Y. Tokura, Phys. Rev. B 66, 180402 (2002).

42 Y. Tomioka, A. Asamitsu, Y. Moritomo, H. Kuwahara, and Y. Tokura, Phys. Rev. Lett. 5108 (1995).

${ }^{43}$ Y. Motome and N. Furukawa, J. Phys. Soc. Japan 68, 3853 (1999).

44 N. Furukawa, Y. Motome, and H. Nakata, Computer Physics Communications 142, 410 (2001).

45 N. Furukawa and Y. Motome, J. Phys. Soc. Jpn. 73, 1482 (2004).

46 Y. Motome and N. Furukawa, Phys. Rev. B 68, 144432 (2003).

47 C. Sen, G. Alvarez, Y. Motome, N. Furukawa, I. A. Sergienko, T. Schulthess, A. Moreo, and E. Dagotto (2005), cond-mat/0509418.

48 Here and in the following sections, Monte Carlo "snapshots" are used to illustrate the prevailing states of the system. although, of course, the Monte Carlo method can only calculate averages. 